\title{
Biometric Fingerprint Implementation for Presence Checking and Room Access Control System
}

\author{
Selamat Muslimin ${ }^{1}$ Yudi Wijanarko ${ }^{1}$ Lucky Indra Kesuma ${ }^{2}$ Renny Maulidda, ${ }^{1, *}$ \\ Yordan Hasan ${ }^{1}$ Abdurrahman ${ }^{1}$ Hasan Basri ${ }^{2}$ \\ ${ }^{1}$ Politeknik Negeri Sriwijaya \\ ${ }^{2}$ Universitas Sriwijaya \\ *Corresponding author. Email: rennymaulidda@polsri.ac.id
}

\begin{abstract}
Nowadays, the utilization of using biometric fingerprint is crucial due to improve the security, data checking and room access control system. The function of biometric fingerprint sensor as control to enter the room, by specific person with access. The development of this system is equipped with student's presence in Electronic Engineering, Politeknik Negeri Sriwijaya, either in classroom or laboratory. The implementation biometric fingerprint certainly helpful in monitoring and recapitulating the presence during teaching learning process because when a fingerprint is detected, data access for presence will be recorded automatically, therefore the classroom or laboratory will safe, effective and efficient. To apply fingerprint biometric technology, a control system was created. The system consists of two main designs are hardware and software design. The hardware components are biometric fingerprint scanner, Arduino board, magnetic door lock, server computer and others. While the software components are developing programs to record, verify and monitor fingerprint data.
\end{abstract}

Keywords: Biometric fingerprint, Presence checking, Access control system, Magnetic lock, Arduino

\section{INTRODUCTION}

The development of technology has a significant impact on people's live, including the field of education. Biometric fingerprint technology is the most widely used system, because it tends to have high degree of accuracy and easy to apply. In general, access control is provision of restrictions for someone to enter certain room and can be used to prevent who does not have access rights [1]. This technology has a fingerprint scanner, electronic device, to scan digital image of fingerprint patterns. Furthermore, these fingerprint biometrics will be integrated with several electronic devices.

Therefore, when the fingerprint scanner verifies the valid fingerprint data, the door opens automatically and the data will be recorded and stored in database of the system [2]. Then, this system is also implemented to the classroom and laboratory for teaching learning activities which making it easier for educational staff to monitor and make presence recapitulation [3].

Some papers have been discussed about attendance/presence/absence system. One presents attendance development with the interfacing of fingerprint sensor, Bluetooth, and LCD encase with in a handy enclosure [4]. Then, research about biometrics attendance checking using principal analysis [5]. Room access control using IoT and Raspberry Pi are done by [6-7].

In this study, we proposed a device that can enhance the security and integrated with course schedule in class room and laboratory, presence checking and monitoring teaching learning activity. Some components are equipped with this system are Arduino board, fingerprint scanner, liquid crystal display, magnetic door lock, relay, PC and software built using Visual Basic as programming language for system working automatically.

\section{REQUIREMENTS REVIEW}

\subsection{Fingerprint}

Fingerprint is an electronic device that applies a scanning sensor to find out a person's fingerprint for identity verification purposes. fingerprint sensors like 
this are used in several electronic equipment such as smartphones, entrances, employee attendance devices and various kinds of electronic equipment that require a high level of security, and can only be accessed by certain people. Before the fingerprint sensor was discovered, data was first secured using a password or ID, some also used a pattern to secure data.

The function of the fingerprint is only one, namely to secure and as a verification medium, just like passwords and patterns, it's just that the verification medium used in fingerprint is a form of human fingerprints, that is what will be used as a medium for ID Primary Key.

The sensor of fingerprint has a high level of sensitivity. The sensor will perform a scanning scan process for each finger that hits the sensor and the scanned data from the sensor will be sent to the computer via USB. The scan process that takes times. After registering for a finger, the data is stored in a database.

A database is a collection if information stored on computer systematic manner and can be checked using computer program to obtain the information from the database. It is arrangement of complete data record of an organization or company which are organized, processed and stored on the computer using certain methods for a specific purpose.

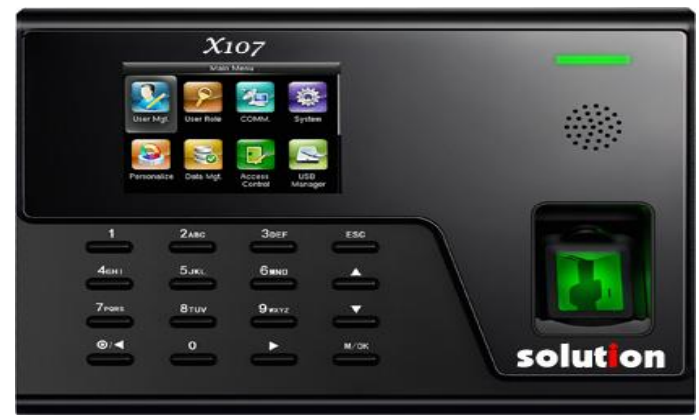

Figure 1 Fingerprint Device

\subsection{Arduino}

Arduino Uno is an open source of electronic kit or circuit board based on the ATmega328 programmable microcontroller. The purpose of embedding the program on it is the electronic circuit can read the input, process it and produce the output. It has flexibility in terms of both hardware and software to facilitate an electronic device in various fields. The default Arduino software includes a serial monitor which is very easy to read and send data. TX and RX indicator LEDs will blink when data via USB to serial connect to USB on the computer.

This microcontroller has 14 digital I/O pins (6 provide PWM output), 6 analog input pins, a $16 \mathrm{MHz}$ clock speed, USB connection, adapter socket, ICSP header pins, and a reset button. It is easily connected with a USB power cable or AC to DC adapter power supply or battery. If using a battery, it can be pinned to the GND and Vin pins in the power connector.

This component board can be supplied with a working voltage of between 6 to 20 volts, if the power supply is below the $5 \mathrm{~V}$ standard, the board will be unstable, if it is forced on the 12 Volt voltage regulator, the Arduino board may overheat and damage the board. It is highly recommended that the voltage is $7-12$ volts.

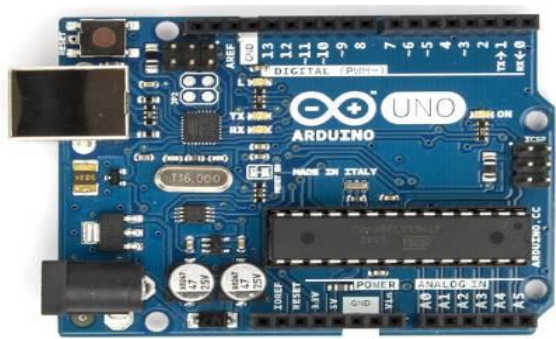

Figure 2 Arduino UNO R3 ATMega328

\subsection{Liquid Crystal Display}

LCD or Liquid Crystal Display is a type of flat media displaying to produce visible images using liquid crystals. Liquid Crystal Display (LCD) technology has been widely used in products such as laptop screens, cellphone screens, calculator screens, digital clock screens, multimeter screens, computer monitors, televisions, portable game screens, digital thermometer screens and other products. other electronic products.

LCD or Liquid Crystal Display basically consists of two main parts, namely the backlight section and the Liquid Crystal section. As mentioned earlier, LCD does not emit any lighting, it only reflects and transmits the light that passes through it.

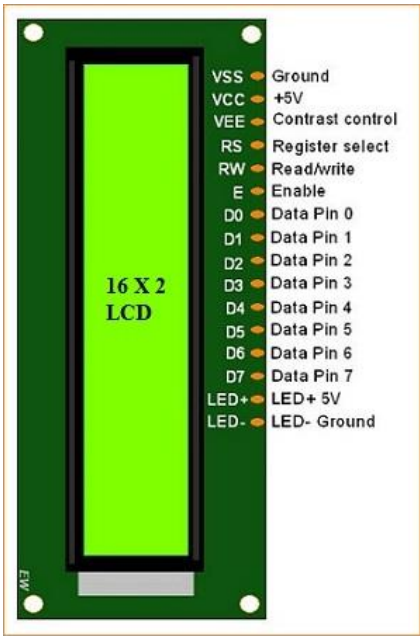

$\underline{L C D-16 \times 2 \text {-pin-diagram }}$

Figure 3 Liquid Crystal Display 


\subsection{Magnetic Door Lock}

One of a type of electronic door lock is magnetic door lock. It is an actuator for locking and unlocking the door in automatic access system. Electronic door lock magnetic type is equipment with very strong magnetic properties which is generated electromagnetically and works as a door lock.

When the electronic door lock magnetic type is applied voltage, it will create a magnet on its surface, become a very strong magnet and the lock will stick to the plate armature. And if there is no voltage then the magnetic properties will disappear.

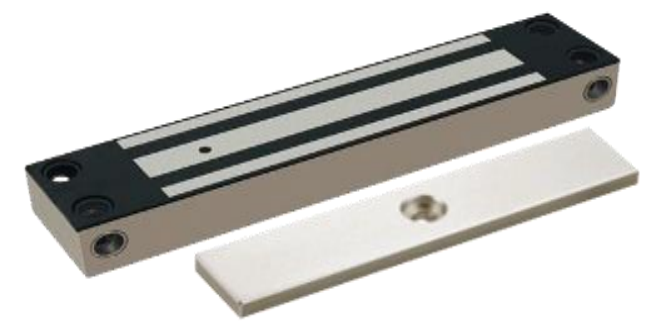

Figure 4 Magnetic Door Lock

\section{METHODOLOGY}

The initial stage of this research is review literatures as the basic of this device. Then, developing the design consist of electronic and software design. Finally, testing of device is necessary in order to get a good final result as expected.

In addition, this design will make it easier to find and repair damaged equipment or circuits. With good design, is obtained in accordance with the wishes of the designer.

\subsection{Electronic Design}

System design objectives is to determine the input, process, on system design to be made, in this stage is electronic design. Electronic design includes input, process (microcontroller), and output. The circuit is an important part of designing a biometric (fingerprint) technology system. The workings of the entire system to be created can be seen in the block diagram so that the entire block diagram will produce a system that can be functioned. Block diagram of the design and analysis of biometric technology systems (fingerprints) as shown in figure below:

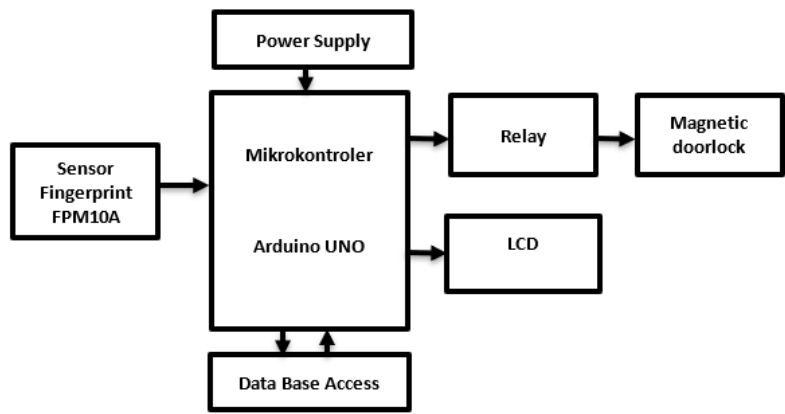

Figure 5 Block Diagram

Electronic design is the main concept of device making. Electronic devices used in this device are Arduino, fingerprint scanner, liquid crystal display, magnetic door lock and relay as shown in figure below:

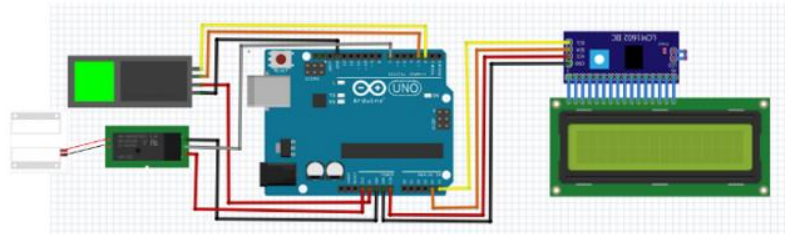

Figure 6 Electronic Circuit

Software design also built using visual basic as programming language for system working automatically. As shown in figure below:

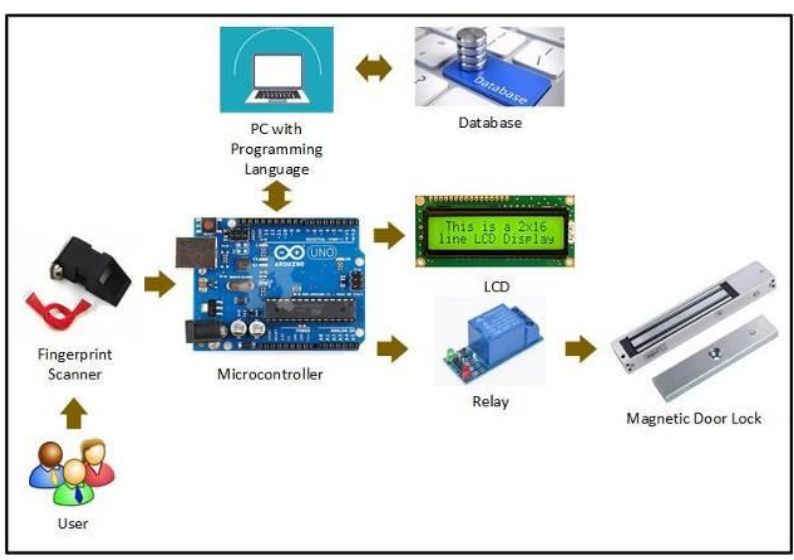

Figure 7 System Architecture

\subsection{Software Design}

The design of a tool requires a performance diagram so that the system can be understood by the flow of how the system works. This system performance diagram is also made to facilitate the manufacture of a designed system. The flow chart of the system can be shown in figure below: 


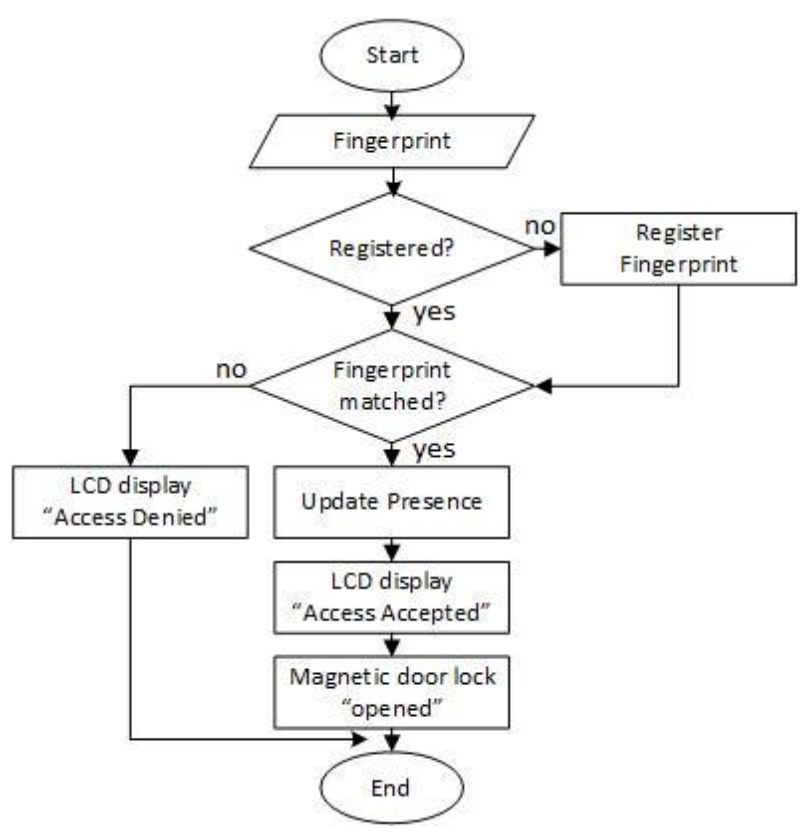

Figure 8 Flow chart system

\section{RESULT AND DISCUSSION}

In this stage, it contains the result display programming in the visual basic application and the construction of data base access as user data and student's presence.

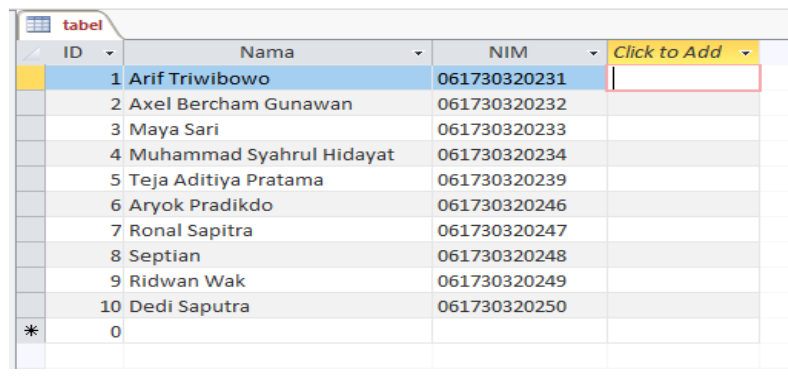

Figure 8 Registered user display

In the fingerprint sensor testing phase, users who have registered on each finger are done by attaching the user's fingerprint to the fingerprint sensor. When the fingerprint successfully verifies the fingerprint, the magnetic door lock will open.

Table 1 Fingerprint testing for registered users

\begin{tabular}{|c|c|c|c|}
\hline Test & Fingerprint & Result & $\begin{array}{c}\text { Magnetic } \\
\text { Doorlock }\end{array}$ \\
\hline First & Thumb & Detected & Open \\
\hline Second & Index finger & Detected & Open \\
\hline Third & Middle finger & Detected & Open \\
\hline Fourth & Ring Finger & Detected & Open \\
\hline Fifth & Little finger & Detected & Open \\
\hline
\end{tabular}

Next is carried out by pasting the recorded fingerprints on the data base in order to find out how long this fingerprint sensor can receive data from the user's fingerprints of the room entrance access right, in this test using two persons, each person input 3 fingers.

Table 2 Fingerprint reading time testing

\begin{tabular}{|c|c|c|}
\hline \multirow{2}{*}{ User } & Fingerprint & Time \\
\cline { 3 - 3 } & Thumb & LCD (Second) \\
\hline \multirow{4}{*}{1} & Index finger & 1,9 \\
\cline { 2 - 3 } & Little finger & 2,1 \\
\hline \multirow{3}{*}{2} & Thumb & 2,4 \\
\cline { 2 - 3 } & Index finger & 1,5 \\
\cline { 2 - 3 } & Little finger & 1,8 \\
\hline
\end{tabular}

This test is carried out by pasting the recorded fingerprints on the data base with the condition of several fingers in damp, dusty, dry and oily conditions. This test is carried out in order to find out some of the state of the finger that can be read or not on the fingerprint sensor reading and can receive data from the user's fingerprint, the access right to the room entrance and as student attendance.

Table 3 Fingerprint sensor readings with several finger

\begin{tabular}{|c|c|c|c|c|c|}
\hline \multirow{2}{*}{ User } & \multirow{2}{*}{ Fingerprint } & \multicolumn{4}{|c|}{ Finger State } \\
\cline { 3 - 6 } & Thumb & Detected & Error & Detected & Detected \\
\hline \multirow{3}{*}{1} & Index & Error & Error & Detected & Detected \\
\cline { 2 - 6 } & Little & Detected & Detected & Detected & Detected \\
\cline { 2 - 6 } & Thumb & Detected & Error & Detected & Detected \\
\hline \multirow{3}{*}{2} & Index & Detected & Error & Detected & Detected \\
\cline { 2 - 6 } & Little & Error & Detected & Detected & Detected \\
\hline
\end{tabular}

In designing a system with fingerprint (biometric) technology, fingerprint functions as sending biometric data to a microcontroller for automatic door openers and as student attendance, initially having to enter fingerprint data on database access through visual basic displays and then test the fingerprint data. The fingerprint sensor will detect the fingerprint and then the translation of the fingerprint will be processed by Arduino. Arduino will send fingerprint data to data base access, if it is true that the fingerprint is registered, a command will be sent to Arduino and Arduino will set the relay to be able to open on, and also the LCD displays a fingerprint display "Access is accepted, please enter".

\section{CONCLUSION}

The device of the biometric fingerprint implementation for presence checking and room access control system can be used to carry out the security and provide process of student's presence recap automatically and computerized in teaching learning activity. Therefore, it easier to control student's presence and improve the security of the classroom and laboratory. 


\section{REFERENCES}

[1] Dexter D. Geralde;Milton M. Manaloto;Dionn Elijah D. Loresca;Jacqueline D. Reynoso;Eden T. Gabion;Gabriel Rodnei M. Geslani, in Microcontroller-based Room Access Control System with Professor Attendance Monitoring using Fingerprint Biometrics technology with Backup Keypad Access System, in: 2017 IEEE 9th International Conference on Humanoid, Nanotechnology, Information Technology, Communication and Control, Environment and Management (HNICEM), IEEE Xplore, Manila, Philippines, 2017, DOI: 10.1109/HNICEM.2017.8269432

[2] Suchit Shavi, Secured room access module, in: 2017 International Conference on Smart technologies For Smart Nation (SmartTechCon), IEEE Xplore, Bengalore, India, 2017. DOI: 10.1109/SmartTechCon.2017.8358546

[3] Rizqia C, Rakhmad A, Efy Y in: Fingerprint for automatic door integrated with absence and user access in: 2016 International Symposium on Electronics and Smart Devices (ISESD), IEEE Xplore, Bandung Indonesia, 2016. DOI: 10.1109/ISESD.2016.7886686

[4] Swarnendu G, Mohammed SKP, Neeraj M, Prabhu KN, Biswajeet C, in: Smart Attendance System in:
2018 International Conference on Smart City and Emerging Technology (ICSCET), IEEE Xplore, Mumbai India, $2018 . \quad$ DOI: 10.1109/ICSCET.2018.8537298

[5] Jennifer C. Dela Cruz, Arnold C. Paglinawan, Miguel Isiah R. Bonifacio, Allan Jake D. Flores, Earl Vic B. Hurna in: Biometrics based attendance checking using Principal Component Analysis in: 2015 IEEE Region 10 Humanitarian Technology Conference (R10-HTC), IEEE Xplore, 2015. DOI: 10.1109/R10-HTC.2015.7391860

[6] B. Ya. Sovetov, T. M. Tatarnikova, V.V. Cehanovsky, in: Physical Access Control System for the Premises using the Internet of Things Technology, in: 2020 9th Mediterranean Conference on Embedded Computing (MECO), IEEE Xplore, 2020 DOI: 10.1109/MECO49872.2020.9134107

[7] M. Taufik, D. Ogi, in: Implementing One-Time Password Mutual Authentication Scheme on Sharing Renewed Finite Random Sub-Passwords Using Raspberry Pi as a Room Access Control to Prevent Replay Attack, in: 2018 International Conference on Electrical Engineering and Informatics (ICELTICs), IEEE Xplore, 2018. DOI: 10.1109/ICELTICS.2018.8548886 